\title{
The Multiresolving Sets of Graphs with Prescribed Multisimilar Equivalence Classes
}

\author{
Varanoot Khemmani iD and Supachoke Isariyapalakul \\ Department of Mathematics, Srinakharinwirot University, Sukhumvit 23, Bangkok 10110, Thailand \\ Correspondence should be addressed to Supachoke Isariyapalakul; supachoke.isa@g.swu.ac.th
}

Received 18 April 2018; Accepted 5 July 2018; Published 1 August 2018

Academic Editor: Dalibor Froncek

Copyright (C) 2018 Varanoot Khemmani and Supachoke Isariyapalakul. This is an open access article distributed under the Creative Commons Attribution License, which permits unrestricted use, distribution, and reproduction in any medium, provided the original work is properly cited.

\begin{abstract}
For a set $W=\left\{w_{1}, w_{2}, \ldots, w_{k}\right\}$ of vertices and a vertex $v$ of a connected graph $G$, the multirepresentation of $v$ with respect to $W$ is the $k$-multiset $\operatorname{mr}(v \mid W)=\left\{d\left(v, w_{1}\right), d\left(v, w_{2}\right), \ldots, d\left(v, w_{k}\right)\right\}$, where $d\left(v, w_{i}\right)$ is the distance between the vertices $v$ and $w_{i}$ for $i=1,2, \ldots, k$. The set $W$ is a multiresolving set of $G$ if every two distinct vertices of $G$ have distinct multirepresentations with respect to $W$. The minimum cardinality of a multiresolving set of $G$ is the multidimension $\operatorname{dim}_{M}(G)$ of $G$. It is shown that, for every pair $k, n$ of integers with $k \geq 3$ and $n \geq 3(k-1)$, there is a connected graph $G$ of order $n$ with $\operatorname{dim}_{M}(G)=k$. For a multiset $\left\{a_{1}, a_{2}, \ldots, a_{k}\right\}$ and an integer $c$, we define $\left\{a_{1}, a_{2}, \ldots, a_{k}\right\}+\{c, c, \ldots, c\}=\left\{a_{1}+c, a_{2}+c, \ldots, a_{k}+c\right\}$. A multisimilar equivalence relation $R_{W}$ on $V(G)$ with respect to $W$ is defined by $u R_{W} v$ if $m r(u \mid W)=m r(v \mid W)+\left\{c_{W}(u, v), c_{W}(u, v), \ldots, c_{W}(u, v)\right\}$ for some integer $c_{W}(u, v)$. We study the relationship between the elements in multirepresentations of vertices that belong to the same multisimilar equivalence class and also establish the upper bound for the cardinality of a multisimilar equivalence class. Moreover, a multiresolving set with prescribed multisimilar equivalence classes is presented.
\end{abstract}

\section{Introduction}

The distance $d(u, v)$ between two vertices $u$ and $v$ in a connected graph $G$ is the length of a shortest $u-v$ path in $G$. For an ordered set $W=\left\{w_{1}, w_{2}, \ldots, w_{k}\right\} \subseteq V(G)$ and a vertex $v$ of $G$, the $k$-vector

$$
r(v \mid W)=\left(d\left(v, w_{1}\right), d\left(v, w_{2}\right), \ldots, d\left(v, w_{k}\right)\right)
$$

is referred to as the representation of $v$ with respect to $W$. The ordered set $W$ is called a resolving set of $G$ if every two distinct vertices of $G$ have distinct representations with respect to $W$. A resolving set of a minimum cardinality is called a minimum resolving set or a basis of $G$ and this cardinality is the dimension $\operatorname{dim}(G)$ of G.

To illustrate these concepts, consider a connected graph $G$ of Figure 1 with $V(G)=\{u, v, w, x, y, z\}$. Considering the ordered set $W_{1}=\{w, z\}$, there are six representations of the vertices of $G$ with respect to $W_{1}$ :

$$
\begin{aligned}
& r\left(u \mid W_{1}\right)=(2,3), \\
& r\left(v \mid W_{1}\right)=(3,2), \\
& r\left(w \mid W_{1}\right)=(0,3), \\
& r\left(x \mid W_{1}\right)=(1,2), \\
& r\left(y \mid W_{1}\right)=(2,1), \\
& r\left(z \mid W_{1}\right)=(3,0) .
\end{aligned}
$$

Since there is no 1-element resolving set of $G$, it follows that $W_{1}$ is a basis of $G$, and so $\operatorname{dim}(G)=2$.

The concepts of resolving sets and minimum resolving sets have previously appeared in [1-4]. Slater in [3, 4] introduced these ideas and used a locating set for what we 


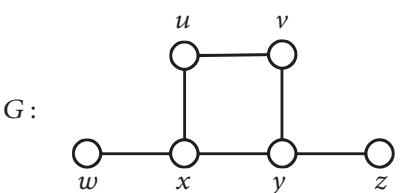

FIGURE 1: A connected graph $G$.

have called a resolving set. He referred to the cardinality of a minimum resolving set in a connected graph as its locating number. He described the usefulness of these ideas when working with US sonar and coast guard LORAN (long range aids to navigation) stations. Harary and Melter [2] discovered these concepts independently as well but used the term metric dimension rather than locating number, the terminology that we have adopted. These concepts were rediscovered by Johnson [5] of the Pharmacia Company while attempting to develop a capability of large datasets of chemical graphs. More applications of these concepts to navigation of robots in networks and other areas are discussed in [6-9].

A multiset is a generalization of the concept of a set, which is like a set except that its members need not to be distinct. For example, the set $\{1,1,2\}$ is the same as the set $\{1,2\}$ but not so for the multiset. The multiset $M=\{5,5,6, a, a, a, a, b, b, b\}$ has 10 elements of 4 different types: 2 of type 5, 1 of type 6,4 of type $a$, and 3 of type $b$. So, the multiset is usually indicated by specifying the number of times different types of elements occur in it. Therefore, the multiset $M$ can be written by $M=\{2 \cdot 5,1 \cdot 6,4 \cdot a, 3 \cdot b\}$. The numbers $2,1,4$, and 3 are called the repetition numbers of the multiset $M$. In particular, a set is a multiset having all repetition numbers equal to 1.

As described in [1], all connected graphs $G$ contain an ordered set $W$ such that each vertex of $G$ is distinguished by a $k$-vector, known as a representation, consisting of its distance from the vertices in $W$. It may also occur that some graph contains a set $W^{\prime}$ with property that the vertices of graph have uniquely distinct $k$-multisets containing their distances from each of the vertices in $W^{\prime}$. The goal of this paper is to study the existence of such a set of connected graphs.

For a set $W=\left\{w_{1}, w_{2}, \ldots, w_{k}\right\}$ of vertices and a vertex $v$ of a connected graph $G$, we refer to the $k$-multiset

$$
m r(v \mid W)=\left\{d\left(v, w_{1}\right), d\left(v, w_{2}\right), \ldots, d\left(v, w_{k}\right)\right\}
$$

as the multirepresentation of $v$ with respect to $W$. The set $W$ is called a multiresolving set of $G$ if every two distinct vertices have distinct multirepresentations with respect to $W$. A multiresolving set of a minimum cardinality is called a minimum multiresolving set or a multibasis of $G$ and this cardinality is the multidimension $\operatorname{dim}_{M}(G)$ of $G$.

For example, consider a connected graph $G$ of Figure 1. As we know $W_{1}=\{w, z\}$ is a basis of $G$. However, $W_{1}$ is not a multiresolving set of $G$ since $m r\left(u \mid W_{1}\right)=\{2,3\}=m r(v \mid$ $\left.W_{1}\right)$. In fact, the set $W_{2}=\{w, x, z\}$ is a multiresolving set of
$G$ with the following multirepresentations of the vertices of $G$ with respect to $W_{2}$ :

$$
\begin{gathered}
m r\left(u \mid W_{2}\right)=\{1,2,3\}, \\
m r\left(v \mid W_{2}\right)=\{2,2,3\}, \\
m r\left(w \mid W_{2}\right)=\{0,1,3\}, \\
m r\left(x \mid W_{2}\right)=\{0,1,2\}, \\
m r\left(y \mid W_{2}\right)=\{1,1,2\}, \\
m r\left(z \mid W_{2}\right)=\{0,2,3\} .
\end{gathered}
$$

It is routine to verify that there are no 1-element and 2element multiresolving sets of $G$. Hence, $W_{2}$ is a multibasis of $G$, and so $\operatorname{dim}_{M}(G)=3$.

Not all connected graphs have a multiresolving set and also $\operatorname{dim}_{M}(G)$ is not defined for all connected graphs $G$. For example, the complete graph $K_{3}$ has no multiresolving set. Thus, $\operatorname{dim}_{M}\left(K_{3}\right)$ is not defined. However, if $G$ is a connected graph of order $n$, for which $\operatorname{dim}_{M}(G)$ is defined, and then every multiresolving set of $G$ is a resolving set of $G$, and so

$$
1 \leq \operatorname{dim}(G) \leq \operatorname{dim}_{M}(G) \leq n .
$$

For every set $W$ of vertices of a connected graph $G$, the vertices of $G$ whose multirepresentations with respect to $W$ contain 0 are vertices in $W$. On the other hand, the multirepresentations of vertices of $G$ which do not belong to $W$ have elements, all of which are positive. In fact, to determine whether a set $W$ is a multiresolving set of $G$, the vertex set $V(G)$ can be partitioned into $W$ and $V(G)-W$ to examine whether the vertices in each subset have distinct multirepresentations with respect to $W$.

The multiresolving set of a connected graph was introduced by Saenpholphat [10] who showed that there is no connected graph $G$ such that $\operatorname{dim}_{M}(G)=2$. Moreover, the multidimensions of complete graphs, paths, cycles, and bipartite graphs were determined. Simanjuntak, Vetrík, and Mulia [11] discovered this concept independently and used a notation $m d(G)$ for a multidimension of a connected graph G.

\section{The Multidimension of a Connected Graph}

Two vertices $u$ and $v$ of a connected graph $G$ are distancesimilar if $d(u, x)=d(v, x)$ for all $x \in V(G)-\{u, v\}$. Certainly, distance similarity in $G$ is an equivalence relation on $V(G)$. For example, consider a complete bipartite graph $K_{r, s}$ with partite sets $U$ and $V$. Every pair of vertices in the same partite set are distance-similar. Then the distance-similar equivalence classes in $K_{r, s}$ are its partite sets $U$ and $V$. The following results were obtained in [10] showing the usefulness of the distance-similar equivalence class to determine the multidimensions of connected graphs.

Theorem 1 (see [10]). Let $G$ be a connected graph such that $\operatorname{dim}_{M}(G)$ is defined. If $U$ is a distance-similar equivalence class in $G$ with $|U|=2$, then every multiresolving set of $G$ contains exactly one vertex of $U$. 
Theorem 2 (see [10]). If $U$ is a distance-similar equivalence class in a connected graph $G$ with $|U| \geq 3$, then $\operatorname{dim}_{M}(G)$ is not defined.

It was shown in $[10,11]$ that a path is the only one of connected graphs with multidimension 1 , and any multiresolving sets of a connected graph cannot contain only two vertices. We state these results in the following theorems.

Theorem 3 (see $[10,11])$. Let $G$ be a connected graph. Then $\operatorname{dim}_{M}(G)=1$ if and only if $G=P_{n}$, the path of order $n$.

Theorem 4 (see $[10,11]$ ). A connected graph has no multiresolving set of cardinality 2 .

Last, we are able to determine all pairs $k, n$ of positive integers with $k \geq 3$ and $n \geq 3(k-1)$ which are realizable as the multidimension and the order of some connected graph. In order to do this, we present an additional notation. For integers $a$ and $b$, let $[a, b]$ be a multiset such that

$$
[a, b]= \begin{cases}\{a, a+1, \ldots, b-1, b\} & \text { if } a<b \\ \{a\} & \text { if } a=b \\ \emptyset & \text { if } a>b\end{cases}
$$

Such a multiset is referred to as a consecutive multiset of integers $a$ and $b$.

Theorem 5. For every pair $k, n$ of integers with $k \geq 3$ and $n \geq 3(k-1)$, there is a connected graph $G$ of order $n$ with $\operatorname{dim}_{M}(G)=k$.

Proof. Let $k$ and $n$ be integers with $k \geq 3$ and $n \geq 3(k-1)$. We consider two cases.

Case $1(n=3(k-1))$. Let $G$ be a graph obtained from the path $P_{k-1}=\left(u_{1}, u_{2}, \ldots, u_{k-1}\right)$ by adding the $2(k-1)$ vertices $v_{i}$ and $w_{i}$ for $1 \leq i \leq k-1$ and joining $v_{i}$ and $w_{i}$ to $u_{i}$, as it is shown in Figure 2. Then the order of $G$ is $n=3(k-1)$. First, we claim that there is no multiresolving set of $G$ with cardinality at most $k-1$. Assume, to the contrary, that there is a multiresolving set $S$ of $G$ such that $|S| \leq k-1$. Since a set $V_{i}=\left\{v_{i}, w_{i}\right\}$ for $1 \leq i \leq k-1$ is a distance-similar equivalence class in $G$, it follows by Theorem 1 that $S$ contains exactly one vertex of $V_{i}$. Without loss of generality, let $w_{i} \in S$ for $1 \leq i \leq k-1$. Thus, $|S|=k-1$. Since $d\left(w_{1}, w_{i}\right)=$ $d\left(w_{k-1}, w_{k-i}\right)$ for all $1 \leq i \leq k-1$, it follows that $m r\left(w_{1} \mid\right.$ $S)=m r\left(w_{k-1} \mid S\right)$ and so a set $S=\left\{w_{1}, w_{2}, \ldots, w_{k-1}\right\}$ is not a multiresolving set of $G$, thereby producing a contradiction. Hence, $\operatorname{dim}_{M}(G) \geq k$. Next, we claim that a set $W=$ $\left\{w_{1}, w_{2}, \ldots, w_{k-1}\right\} \cup\left\{u_{1}\right\}$ is a multiresolving set of $G$. For a vertex $x \in W$, the multirepresentation of $x$ with respect to $W$ is

$$
\begin{aligned}
& m r(x \mid W) \\
& = \begin{cases}\{0, i\} \cup[3, i+1] \cup[3, k-i+1] & \text { if } x=w_{i} \quad(1 \leq i \leq k-1) \\
{[0, k-1]} & \text { if } x=u_{1} .\end{cases}
\end{aligned}
$$

For $2 \leq i \leq k-1$, the multirepresentation of $u_{i}$ with respect to $W$ is

$$
m r\left(u_{i} \mid W\right)=\{1, i-1\} \cup[2, i] \cup[2, k-i] .
$$

For $1 \leq i \leq k-1$, the multirepresentation of $v_{i}$ with respect to $W$ is

$$
m r\left(v_{i} \mid W\right)=\{2, i\} \cup[3, i+1] \cup[3, k-i+1] .
$$

Therefore, $W$ is a multiresolving set of $G$ with $|W|=k$. Hence, $\operatorname{dim}_{M}(G)=k$.

Case $2(n>3(k-1))$. Let $H$ be a graph obtained from the graph $G$ in Case 1 by adding the path $P=$ $\left(x_{1}, x_{2}, \ldots, x_{n-3(k-1)}\right)$ and joining $x_{1}$ to $v_{k-1}$ and $w_{k-1}$, as it is shown in Figure 3. By a similar argument to the one used in Case 1 , it is shown that there is no $l$-multiresolving set of $H$ with $1 \leq l \leq k-1$. We claim that a set $W=\left\{w_{1}, w_{2}, \ldots, w_{k-1}\right\} \cup$ $\left\{u_{1}\right\}$ is a multiresolving set of $H$. For vertices in $V(H)-$ $\left\{x_{1}, x_{2}, \ldots, x_{n-3(k-1)}\right\}$, their multirepresentations with respect to $W$ are the same as in Case 1 . For $1 \leq i \leq n-3(k-1)$, the multirepresentation of $x_{i}$ with respect to $W$ is

$$
m r\left(x_{i} \mid W\right)=\{i, i+k-1\} \cup[i+3, i+k] .
$$

Hence, $W$ is a multiresolving set of $H$ with $|W|=k$, and so $\operatorname{dim}_{M}(H)=k$.

\section{Multisimilar Equivalence Relation}

In this section, we investigate another equivalence relation on a vertex set of a connected graph. First, we need some additional definitions and notations. Let $A=\left\{\left\{a_{1}, a_{2}, \ldots, a_{k}\right\}\right.$ | $a_{i} \in \mathbb{Z}$ for $\left.1 \leq i \leq k\right\}$ be a collection of multisets. For an integer $c$, we define

$$
\begin{aligned}
& \left\{a_{1}, a_{2}, \ldots, a_{k}\right\}+\{c, c, \ldots, c\} \\
& \quad=\left\{a_{1}+c, a_{2}+c, \ldots, a_{k}+c\right\}
\end{aligned}
$$

where $\left\{a_{1}, a_{2}, \ldots, a_{k}\right\} \in A$. Let $W$ be a set of vertices of a connected graph $G$ and let $u$ and $v$ be vertices of $G$. A multisimilar relation $R_{W}$ with respect to $W$ on a vertex set $V(G)$ is defined by $u R_{W} v$ if there is an integer $c_{W}(u, v)$ such that

$$
\begin{aligned}
m r(u \mid W)= & m r(v \mid W) \\
& +\left\{c_{W}(u, v), c_{W}(u, v), \ldots, c_{W}(u, v)\right\} .
\end{aligned}
$$

An integer $c_{W}(u, v)$ satisfying (12) is called a multisimilar constant of $u R_{W} v$ or simply a multisimilar constant. Clearly, $R_{W}$ is an equivalence relation on $V(G)$. For each vertex $u$ in $V(G)$, let $[u]_{W}$ denote the multisimilar equivalence class of $u$ with respect to $W$. Then 


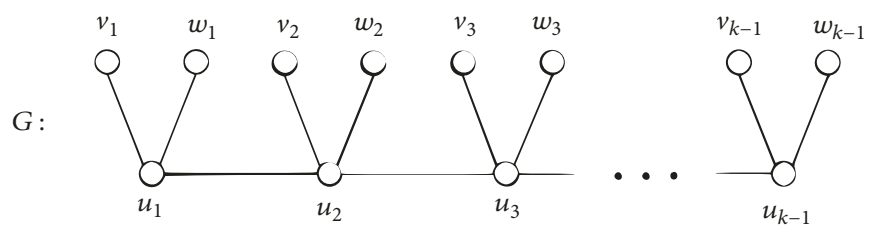

Figure 2: A connected graph $G$ in Case 1.

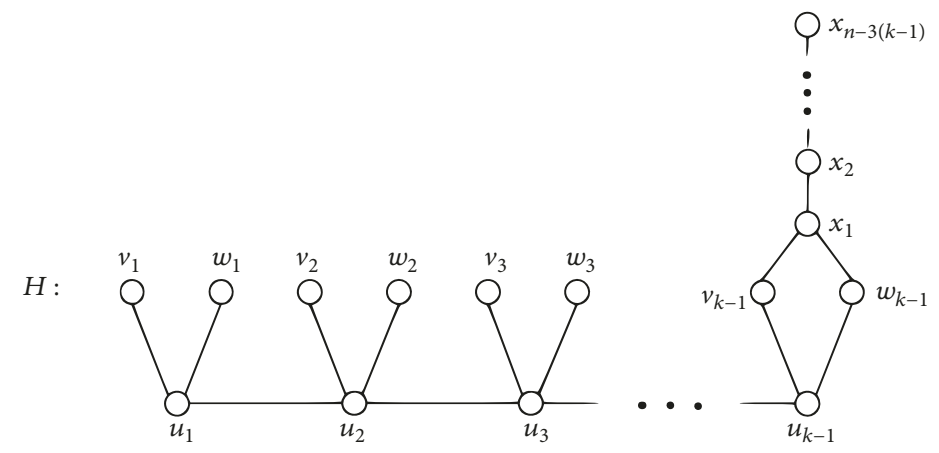

FIgURE 3: A connected graph $H$ in Case 2.

$x \in[u]_{W} \quad$ if and only if $m r(x \mid W)=m r(u \mid W)+\left\{c_{W}(x, u), c_{W}(x, u), \ldots, c_{W}(x, u)\right\}$,

where $c_{W}(x, u)$ is a multisimilar constant. Observe that if $x \in[u]_{W}$, then there is a multisimilar constant $c_{W}(x, u)$ with a property that, for every vertex $w \in W$, there is a corresponding vertex $w^{\prime} \in W$ such that

$$
d(x, w)=d\left(u, w^{\prime}\right)+c_{W}(x, u)
$$

With this observation, we may as well say that $x \in[u]_{W}$ if and only if there are multisimilar constant $c_{W}(x, u)$ and a bijective function $f$ on $W$ defined as

$$
\begin{aligned}
& f(w)=w^{\prime} \\
& \quad \text { whenever } d(x, w)=d\left(u, w^{\prime}\right)+c_{W}(x, u) .
\end{aligned}
$$

The function $f$ is called a multisimilar function of $x R_{W} u$ or a multisimilar function if there is no ambiguity. Consequently, it is not surprising that an inverse function $f^{-1}$ is also multisimilar function of $v R_{W} u$ with a multisimilar constant $c_{W}(v, u)=-c_{W}(u, v)$.

To illustrate these concepts, consider a vertex $u$ in a connected graph $G$ of Figure 1 and the set $W=\{w, y, z\}$. There is only one vertex $x$ in $V(G)-\{u\}$ such that $x$ is related to $u$ by a multisimilar relation $R_{W}$ with a multisimilar constant $c_{W}(x, u)=-1$; that is,

$$
m r(x \mid W)=m r(u \mid W)+\{-1,-1,-1\} .
$$

Therefore, $[u]_{W}=\{u, x\}$. Thus, a multisimilar function $f$ of $x R_{W} u$ is defined by

$$
\begin{aligned}
f(w) & =y, \\
f(y) & =w \\
\text { and } f(z) & =z .
\end{aligned}
$$

Moreover, there is another multisimilar function $f^{\prime}$ of $x R_{W} u$; that is,

$$
\begin{aligned}
f^{\prime}(w) & =w, \\
f^{\prime}(y) & =y \\
\text { and } f^{\prime}(z) & =z .
\end{aligned}
$$

The example just described shows an important point that the multisimilar function of any two vertices in the same multisimilar equivalence class with respect to a set $W$ is not necessarily unique.

More generally, for a vertex $u$ and a set $W$ of vertices of a connected graph $G$, let $m r(u \mid W)=\left\{r_{1} \cdot a_{1}, r_{2} \cdot a_{2}, \ldots, r_{l} \cdot a_{l}\right\}$, where $a_{1}<a_{2}<\cdots<a_{l}$ and $r_{i}$ is a repetition number of type $a_{i}$ for each $i$ with $1 \leq i \leq l$. If $u \in[v]_{W}$, where $v \in V(G)$, then it follows by (13) and (14) that, for each type of $m r(u \mid W)$, there is a corresponding type of $m r(v \mid W)$ such that their repetition numbers are equal. Therefore, we may assume that $m r(v \mid W)=\left\{r_{1} \cdot b_{1}, r_{2} \cdot b_{2}, \ldots, r_{l} \cdot b_{l}\right\}$, where $b_{1}<b_{2}<\cdots<b_{l}$. For each integer $i$ with $1 \leq i \leq l$, let $A_{i}=\{w \in W \mid d(u, w)=$ 
$\left.a_{i}\right\}$ and $B_{i}=\left\{w \in W \mid d(v, w)=b_{i}\right\}$. Then the types of $m r(u \mid$ $W)$ partition $W$ into $l$ sets $A_{1}, A_{2}, \ldots, A_{l}$. On the other hand, $W$ is also partitioned into $l$ sets $B_{1}, B_{2}, \ldots, B_{l}$ depending on the types of $m r(v \mid W)$. Hence, the multisimilar function $f$ of $u R_{W} v$ has the property that, for every vertex $w \in A_{i}$, there is a vertex $w^{\prime} \in B_{i}$ such that

$$
f(w)=w^{\prime}
$$

where $1 \leq i \leq l$. Indeed, there are $r_{1} ! r_{2} ! \cdots r_{l} !$ distinct multisimilar functions of $u R_{W} v$. These observations yield the following result.

Theorem 6. Let $W$ be a set of vertices of a connected graph $G$ and let $u$ and $v$ be vertices of $G$ such that $u \in[v]_{W}$. Suppose that $\operatorname{mr}(u \mid W)=\left\{r_{1} \cdot a_{1}, r_{2} \cdot a_{2}, \ldots, r_{l} \cdot a_{l}\right\}$, where $a_{1}<a_{2}<\cdots<a_{l}$ and $r_{i}$ is a repetition number of type $a_{i}$ for each $i$ with $1 \leq i \leq l$. Then

(i) $m r(v \mid W)=\left\{r_{1} \cdot b_{1}, r_{2} \cdot b_{2}, \ldots, r_{l} \cdot b_{l}\right\}$ for some integers $b_{1}, b_{2}, \ldots, b_{l}$ with $b_{1}<b_{2}<\cdots<b_{l}$,

(ii) there is a multisimilar function $f$ of $u R_{W} v$ such that $f\left(w_{i}\right)=w_{i}^{\prime}$, where $d\left(u, w_{i}\right)=a_{i}$ and $d\left(v, w_{i}^{\prime}\right)=b_{i}$ for each $i$ with $1 \leq i \leq l$,

(iii) there are $r_{1} ! r_{2} ! \cdots r_{l}$ ! distinct multisimilar functions of $u R_{W} v$.

By Theorem 6, the following result is obtained.

Corollary 7. Let $W$ be a set of vertices of a connected graph $G$ and let $u$ and $v$ be vertices of $G$ such that $u \in[v]_{W}$ with a multisimilar constant $c_{W}(u, v)$. Then

(i) if $M_{1}$ and $M_{2}$ are the maximum elements of $m r(u \mid W)$ and $m r(v \mid W)$, respectively, then $M_{1}=M_{2}+c_{W}(u, v)$,

(ii) if $m_{1}$ and $m_{2}$ are the minimum elements of $m r(u \mid W)$ and $m r(v \mid W)$, respectively, then $m_{1}=m_{2}+c_{W}(u, v)$.

Proof. Suppose that $u \in[v]_{W}$. Let $\operatorname{mr}(u \mid W)=\left\{r_{1} \cdot a_{1}, r_{2}\right.$. $\left.a_{2}, \ldots, r_{l} \cdot a_{l}\right\}$ and $m r(v \mid W)=\left\{r_{1} \cdot b_{1}, r_{2} \cdot b_{2}, \ldots, r_{l} \cdot b_{l}\right\}$, where $a_{1}<a_{2}<\cdots<a_{l}$ and $b_{1}<b_{2}<\cdots<b_{l}$. Since $M_{1}$ and $M_{2}$ are the maximum elements of $m r(u \mid W)$ and $m r(v \mid W)$, respectively, there are vertices $w$ and $w^{\prime}$ in $W$ such that $M_{1}=d(u, w)=a_{l}$ and $M_{2}=d\left(v, w^{\prime}\right)=b_{l}$. It follows by Theorem 6 that there is a multisimilar function $f$ of $u R_{W} v$ such that $f(w)=w^{\prime}$. Then $d(u, w)=d\left(v, w^{\prime}\right)+c_{W}(u, v)$, where $c_{W}(u, v)$ is a multisimilar constant. Thus, (i) holds. For (ii), the statement may be proven in the same way as (i), and therefore such proof is omitted.

Next, we are prepared to establish the upper bound for the cardinality of a multisimilar equivalence class of a vertex in a connected graph. To show this, let us present a useful proposition as follows.

Proposition 8. Let $W$ be a set of vertices of a connected graph $G$ and let $u$ and $v$ be vertices of $G$ such that $u \in[v]_{W}$. Then $\operatorname{mr}(u \mid W)$ and $\operatorname{mr}(v \mid W)$ have the same minimum (or maximum) element if and only if $m r(u \mid W)=m r(v \mid W)$.
Proof. If $m r(u \mid W)=m r(v \mid W)$, then the minimum (and maximum) elements of $m r(u \mid W)$ and $m r(v \mid W)$ are the same. For the converse, assume that $m_{1}$ and $m_{2}$ are the minimum elements of $m r(u \mid W)$ and $m r(v \mid W)$, respectively, such that $m_{1}=m_{2}$. Since $u \in[v]_{W}$, there is a multisimilar constant $c_{W}(u, v)$ such that

$$
\begin{aligned}
m r(u \mid W)= & m r(v \mid W) \\
& +\left\{c_{W}(u, v), c_{W}(u, v), \ldots, c_{W}(u, v)\right\} .
\end{aligned}
$$

By Corollary 7 (ii), it follows that $m_{1}=m_{2}+c_{W}(u, v)$. Thus, $c_{W}(u, v)=0$. Hence, $m r(u \mid W)=m r(v \mid W)$. Similarly, if $m r(u \mid W)$ and $m r(v \mid W)$ have the same maximum element, then $m r(u \mid W)=m r(v \mid W)$.

Theorem 9. If $W$ is a multiresolving set of a connected graph $G$, then the cardinality of multisimilar equivalence class of each vertex of $G$ with respect to $W$ is at most $\operatorname{diam}(G)+1$.

Proof. Assume, to the contrary, that there is a vertex $v$ of $G$ such that $[v]_{W}$ has the cardinality at least $\operatorname{diam}(G)+2$. Since the minimum elements of multirepresentations of vertices in $[v]_{W}$ with respect to $W$ have at most $\operatorname{diam}(G)+1$ distinct values, there are at least two vertices $x$ and $y$ in $[v]_{W}$ having the same value of the minimum element of $\operatorname{mr}(x \mid W)$ and $m r(y \mid W)$. It follows by Proposition 8 that $m r(x \mid W)=$ $m r(y \mid W)$, contradicting the fact that $W$ is a multiresolving set of $G$.

We can show that the upper bound in Theorem 9 is sharp. Consider the path $P_{n}=\left(v_{1}, v_{2}, \ldots, v_{n}\right)$. We have that $\operatorname{diam}\left(P_{n}\right)=n-1$ and the set $W=\left\{v_{1}\right\}$ is a multiresolving set of $P_{n}$. Thus, $\left[v_{1}\right]_{W}$ contains all vertices of $P_{n}$, and so $\left|\left[v_{1}\right]_{W}\right|=n$.

In the last result, we describe the properties of a multisimilar equivalence classes with respect to a set of vertices.

Theorem 10. Let $u$ and $v$ be vertices of a connected graph $G$ and let $W$ be a set of vertices of $G$. Then

(i) if $[u]_{W} \neq[v]_{W}$, then $\operatorname{mr}(x \mid W) \neq m r(y \mid W)$ for all $x \in[u]_{W}$ and $y \in[v]_{W}$,

(ii) if $[u]_{W}=\{u\}$ for all $u \in V(G)$, then $W$ is a multiresolving set of $G$.

Proof. (i) Assume, to the contrary, that there exist two distinct vertices $x \in[u]_{W}$ and $y \in[v]_{W}$ such that $\operatorname{mr}(x \mid W)=$ $m r(y \mid W)$. Then there are multisimilar constants $c_{W}(x, u)$ and $c_{W}(y, v)$ such that $m r(x \mid W)=\operatorname{mr}(u \mid W)+$ $\left\{c_{W}(x, u), c_{W}(x, u), \ldots, c_{W}(x, u)\right\}$ and $m r(y \mid W)=m r(v \mid$ $W)+\left\{c_{W}(y, v), c_{W}(y, v), \ldots, c_{W}(y, v)\right\}$. Therefore,

$$
\begin{aligned}
m r(u \mid W)+\left\{c_{W}(x, u), c_{W}(x, u), \ldots, c_{W}(x, u)\right\} \\
=m r(v \mid W) \\
\quad+\left\{c_{W}(y, v), c_{W}(\mathrm{y}, v), \ldots, c_{W}(y, v)\right\} .
\end{aligned}
$$

Thus, $m r(u \mid W)=m r(v \mid W)+\left\{c_{W}(y, v)-c_{W}(x, u), c_{W}(y, v)-\right.$ $\left.c_{W}(x, u), \ldots, c_{W}(y, v)-c_{W}(x, u)\right\}$. Hence, $u$ belongs to $[v]_{W}$, which is a contradiction. 
(ii) Assume, to the contrary, that $W$ is not a multiresolving set of $G$. Then there exist two distinct vertices $x$ and $y$ such that $\operatorname{mr}(x \mid W)=\operatorname{mr}(y \mid W)$. Hence, $y$ belongs to $[x]_{W}$, producing a contradiction.

\section{Final Remarks}

The complete graph $K_{n}$ is only one graph that its dimension is $n-1$ but not so for multidimensions. It follows by $[10,11]$ that the multidimension of complete graph is not defined. Thus, (5) leads us to the conjecture:

If $G$ is a connected graph such that $\operatorname{dim}_{M}(G)$ is defined, then $\operatorname{dim}_{M}(G) \leq n-2$.

\section{Data Availability}

No data sharing was used to support this study as no datasets were generated or analyzed during the current study. Other data sources are referenced throughout the paper.

\section{Conflicts of Interest}

The authors declare that they have no conflicts of interest.

\section{Acknowledgments}

This work was funded by the Faculty of Science, Srinakharinwirot University.

\section{References}

[1] G. Chartrand, L. Eroh, M. A. Johnson, and O. R. Oellermann, "Resolvability in graphs and the metric dimension of a graph," Discrete Applied Mathematics, vol. 105, no. 1-3, pp. 99-113, 2000.

[2] F. Harary and R. A. Melter, "On the metric dimension of a graph," Ars Combinatoria, vol. 2, pp. 191-195, 1976.

[3] P. J. Slater, "Leaves of trees," Congressus Numerantium, vol. 14, pp. 549-559, 1975.

[4] P. J. Slater, "Dominating and reference sets in a graph," Journal of Mathematical and Physical Sciences, vol. 22, no. 4, pp. 445-455, 1988.

[5] M. Johnson, "Browsable structure-activity datasets," in Advances in Molecular Similarity Volume 2, vol. 2 of Advances in Molecular Similarity, pp. 153-170, Elsevier, 1999.

[6] B. L. Hulme, A. W. Shiver, and P. J. Slater, FIRE, a subroutine for fire protection network analysis (SAND 81-1261), Sandia National Laboratories, New Mexico, 1981.

[7] B. L. Hulme, A. W. Shiver, and P. J. Slater, Computing minimum cost fire protection (SAND 820809, Sandia National Laboratories, New Mexico, 1982.

[8] B. L. Hulme, A. W. Shiver, and P. J. Slater, "A Boolean Algebraic Analysis of Fire Protection," North-Holland Mathematics Studies, vol. 95, no. C, pp. 215-227, 1984.

[9] S. Khuller, B. Rsghavachari, and A. Rosenfeld, Localization in graphs (CS-TR-3326), University of Maryland, Maryland, 1994.

[10] V. Saenpholphat, "On multiset dimension in graphs," Academic SWU, vol. 1, pp. 193-202, 2009.

[11] R. Simanjuntak, T. Vetrík, and P. B. Mulia, "The multiset dimension of graphs," Discrete Applied Mathematics. 


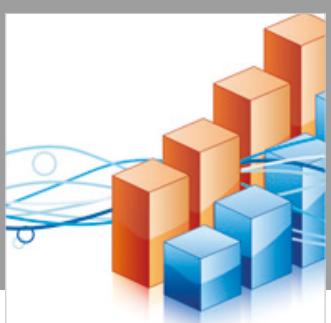

Advances in

Operations Research

\section{-n-m}
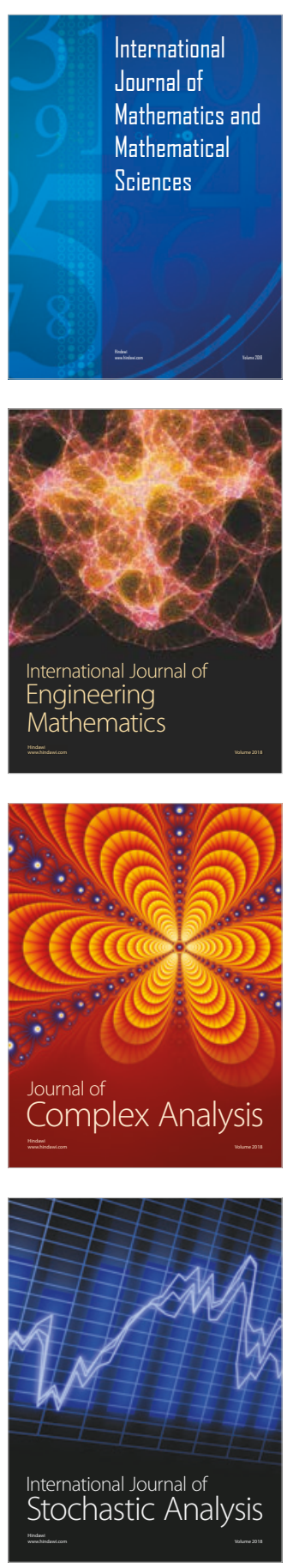
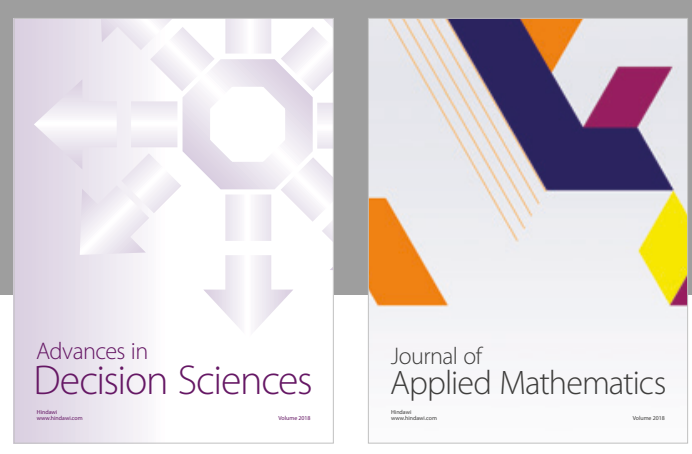

Journal of

Applied Mathematics
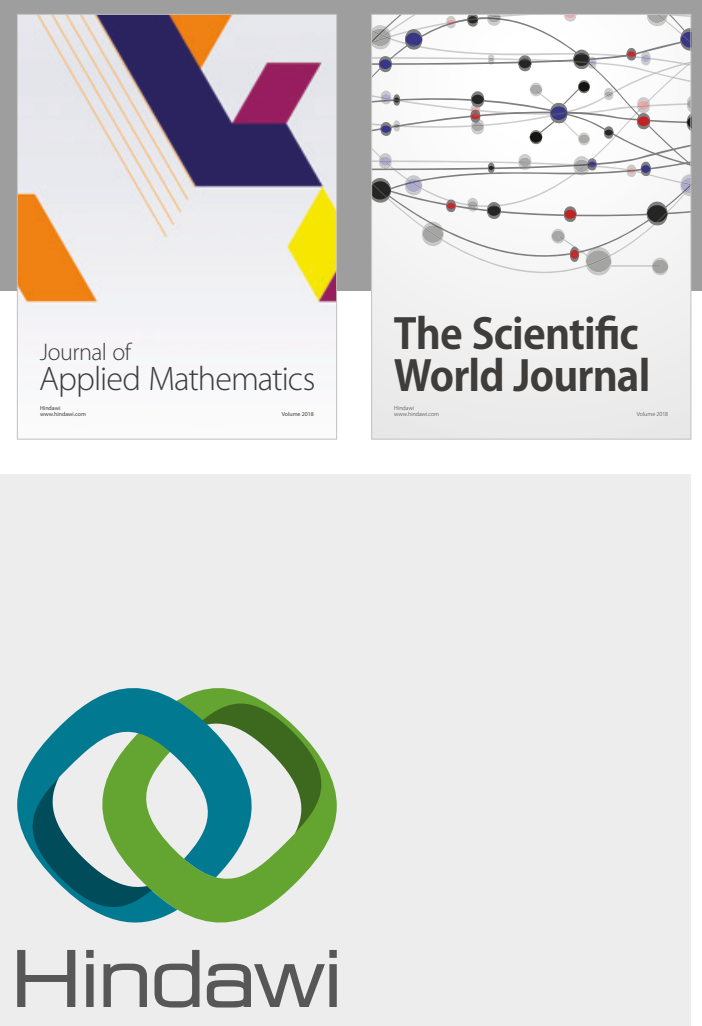

Submit your manuscripts at

www.hindawi.com

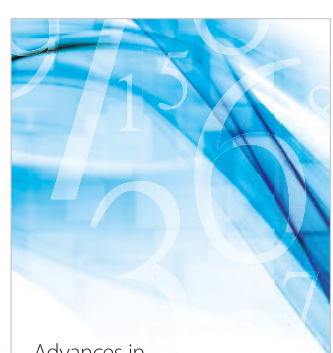

Advances in
Numerical Analysis
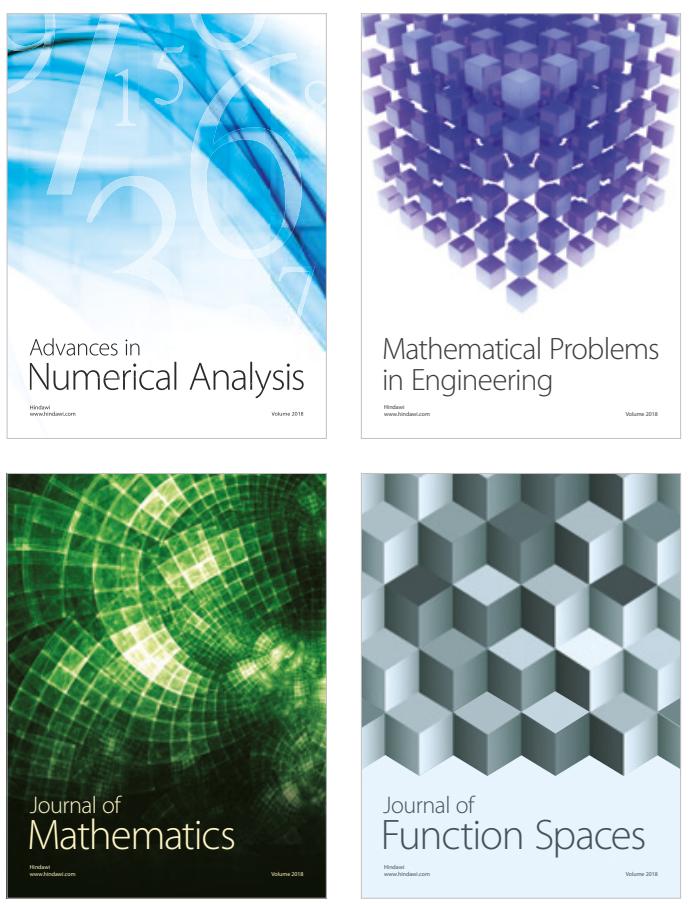

Mathematical Problems in Engineering

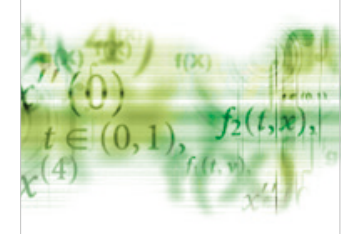

International Journal of

Differential Equations

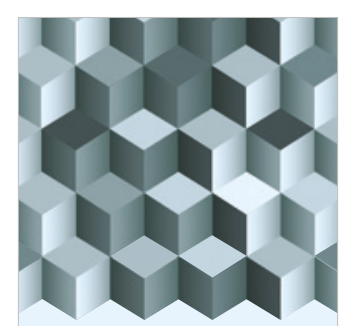

Journal of

Function Spaces

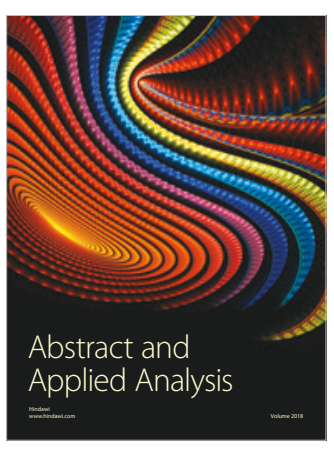

The Scientific

World Journal

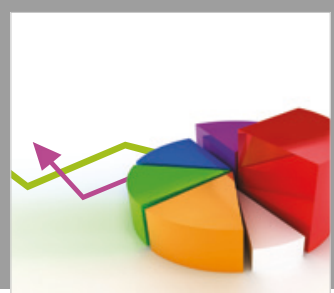

Journal of

Probability and Statistics
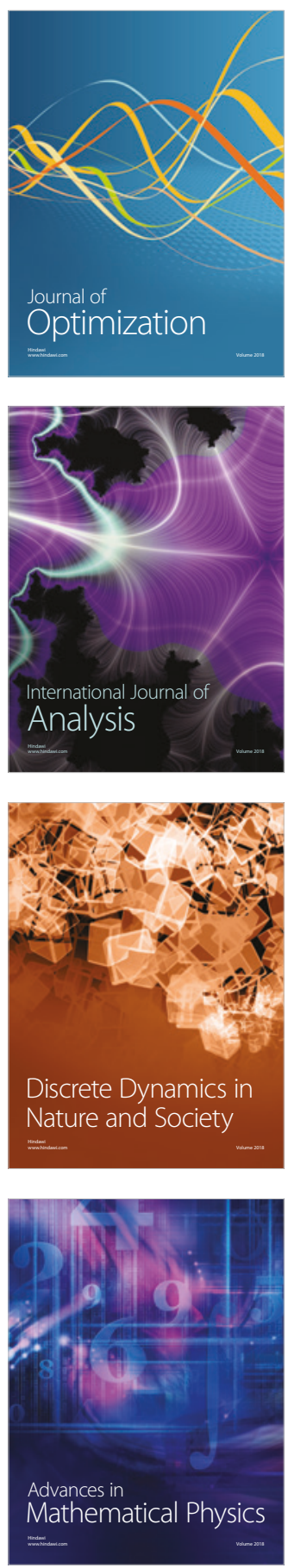